Disponível em:

http://editora.unoesc.edu.br/index.php/race

Race, Joaçaba, v. 14, n. 1, p. 11-38, jan./abr. 2015

\title{
A QUALIDADE DAS INFORMAÇÕES PRESTADAS PELAS EMPRESAS REDUZ OS RISCOS DE INVESTIMENTO? UMA ANÁLISE EMPÍRICA PARA OS DIFERENTES NÍVEIS DE GOVERNANÇA CORPORATIVA DAS EMPRESAS BRASILEIRAS
}

The quality of information provided by reduces business investment risks? an empirical analysis for the governance of different levels of corporate brazilian

Cássio Nóbrega Besarria

E-mail: cassiodanobrega@yahoo.com.br Doutor em Economia pela Universidade Federal de Pernambuco; Professor do Curso de Economia na Universidade Federal de Pernambuco; Endereço: Avenida Professor Morais Rego, 1235, Cidade Universitária, 50670-901, Recife, Pernambuco, Brasil.

Ângelo Antonio Paula

E-mail: angeloantonio198@gmail.com Graduado em Economia pela Universidade Federal de Pernambuco.

Breno Silva Araújo E-mail: brenosapereira@hotmail.com Graduado em Economia pela Universidade Federal de Pernambuco.

José Neto Alves

E-mail: netoalves.barbosa@hotmail.com Graduado em Economia pela Universidade Federal de Pernambuco.

Fenelon Francisco Almeida

E-mail: almeida.fenelon@gmail.com Graduado em Economia pela Universidade Federal de Pernambuco.

Valdeir Soares Monteiro

E-mail: valdeir47@hotmail.com Graduado em Economia pela Universidade Federal de Pernambuco. 
Resumo

O objetivo geral deste estudo consistiu em verificar se a qualidade das informações prestadas pelas empresas e a ampliação dos direitos societários reduzem os riscos de investimento. Para se alcançar o objetivo, esta pesquisa se divide em duas etapas: no primeiro momento, serão formadas três carteiras de investimento de variância mínima, em que as empresas serão classificadas de acordo com diferentes níveis de governança corporativa: Nível 1 (N1), Nível 2 (N2) e Novo Mercado (NM). E será estimado o modelo de precificação de ativos conhecido como versão condicional do Capital Asset Pricing Model (CAPM) de Sharpe-Lintner. Por meio desse modelo será possível obter a relação risco/retorno existente entre as carteiras de investimento e a carteira de mercado, bem como as manifestações do agrupamento da volatilidade. Entre os resultados obtidos, destaca-se que por meio das estimativas das volatilidades foi possível comprovar que as ações das empresas do Novo Mercado são as que apresentam menor risco, seguidas pelas ações classificadas no Nível 2 e no Nível 1, respectivamente. Esses resultados reforçam a hipótese de que a transparência, a qualidade das informações prestadas pelas empresas e a ampliação dos direitos societários reduzem os riscos de investimento.

Palavras-chave: Governança corporativa. Volatilidade condicional. Risco.

\section{The quality of information provided by reduces business investment risks? an empirical analysis for the governance of different levels of corporate brazilian}

\section{Abstract}

The general goal of the present study was to asses if the quality of information provided by firms and the expansion of societal rights reduce investment risks. To achieve this goal, the present research is going to be divided into two steps: first, three minimum variance investment portfolios will be formed, where firms will be classified according to different levels of corporative governance: firms of the Level 1 of governance (N1), firms of the Level 2 of governance (N2) and firms of the New Market (NM). It will be estimated the Capital Asset Pricing Model $(C A P M)$ in conditional version, also known as Sharpe-Linter CAPM conditional version. Through this model it will be possible to obtain the risk/return relation that exists between investment portfolios and market portfolio, as well as manifestations in the volatility in the group. Among the results, it is noteworthy that by the estimates of volatilities was possible to prove that the actions of the New Market companies are those with lower risk, followed by shares classified in Level 2 and Level 1, respectively. These results support the hypothesis that transparency, quality of information provided by companies and the expansion of corporate rights reduce investment risks. Keywords: Corporate governance. Conditional volatility. Risk.

\section{INTRODUÇÃO}

A premissa básica da teoria dos mercados eficientes é que os preços de mercado das ações refletem o conjunto de informações disponíveis e apresentam 
grande sensibilidade a novos dados. Esses conceitos se materializam na melhoria da qualidade das informações prestadas pelas companhias aos seus acionistas e na ampliação dos direitos societários, reduzindo as incertezas no processo de avaliação e de investimento e, consequentemente, o risco, possibilitando, segundo Malacrida e Yamamoto (2006), maior grau de credibilidade e segurança ao se escolher uma alternativa de investimento em detrimento de outras possíveis.

Nesse sentido, a literatura documenta que as boas práticas de governança corporativa passam a desempenhar um papel relevante na redução do risco, visto que empresas listadas nesses segmentos se comprometem a realizar melhorias nas práticas de governança corporativa, ampliando os direitos dos acionistas minoritários e aumentando a transparência das informações divulgadas ao mercado.

No Brasil, a adoção dos princípios e práticas da boa Governança Corporativa teve início na década de 1990 com a criação do Instituto Brasileiro de Conselheiros de Administração (IBCA) que, posteriormente, ficou conhecido como Instituto Brasileiro de Governança Corporativa (IBGC). Segundo Monte et al. (2010), esse instituto foi criado com o propósito de cooperar com o aprimoramento do padrão de governo das empresas nacionais, para seu sucesso e perpetuação.

Posteriormente, a Bolsa de Valores de São Paulo decidiu criar os níveis de Governança Corporativa para as empresas que negociam nesse mercado; os segmentos criados se dividem em Novo Mercado, Nível 2 e Nível 1, criando um ambiente de negociações propício para estimular o interesse dos investidores e a valorização das companhias.

Baseada nessa discussão, esta pesquisa teve o propósito de verificar se a qualidade das informações prestadas pelas empresas e a ampliação dos direitos societários reduzem os riscos de investimento. Com isso, procura-se avaliar os efeitos dos níveis diferenciados de governança corporativa sobre o risco ou a volatilidade das carteiras de investimento, no período de 04 de janeiro de 2010 a 17 de janeiro de 2014. Em relação à escolha do período de análise, esta foi dada pela disponibilidade dos dados para as ações classificadas no Nível de governança N2. Por fim, destacase que a contribuição deste trabalho é essencialmente empírica, apresentando fatos estilizados que ajudem no entendimento dos efeitos das boas práticas de governança corporativa sobre um importante indicador de tomada de decisão de investimento que é o risco.

Em relação aos trabalhos aplicados à economia brasileira e que tratam dessa temática, destaca-se o trabalho desenvolvido por Kalatzis, Sirqueira e Toledo (2008), no qual esses autores procuraram verificar se uma carteira formada com ações de 
empresas que adotam práticas de governança corporativa proporciona maior retorno ao investidor do que uma carteira formada por ações de empresas que não adotam esse conjunto de medidas. $\mathrm{O}$ método adotado na análise foi baseado na teoria de otimização linear e em dois modelos de programação linear. Os resultados obtidos por esses autores sugeriram diferença entre os retornos fornecidos por essas carteiras, mostrando que as empresas que adotaram os princípios de governança corporativa proporcionam maior retorno do que as empresas que não implementaram essas práticas.

Por outro lado, Rogers, Machado Filho e Securato (2008) utilizaram o modelo autorregressivo com heterocedasticidade generalizada (GARCH) e concluíram que empresas que não seguem as diretrizes de governança corporativa apresentam maior tendência de reação ao mercado, isto é, reagem com mais frequência às mudanças no mercado e possuem maior assimetria de informação. Já as empresas com alto grau de governança possuem maior persistência de suas volatilidades e em longo prazo as empresas de maior nível de governança tendem a reduzir suas volatilidades.

$\mathrm{Na}$ mesma linha de argumentação proposta pelo trabalho anterior, Monte et al. (2010) procuraram analisar a composição, o risco e a persistência da volatilidade de três carteiras de variância mínima formadas, respectivamente, por ativos de empresas que fazem parte dos níveis de governança Nível 1 (N1), Nível 2 (N2) e Novo Mercado (NM). Os resultados obtidos por esses autores revelaram que, por meio da análise da formação de carteiras, foi confirmada a tese de menor persistência de volatilidade nas empresas do Novo Mercado, ou seja, os efeitos de um choque negativo (cenários econômicos pessimistas) sobre a volatilidade dessa carteira levarão menos tempo para desaparecer. E concluem que a carteira formada por ativos de empresas do Novo Mercado é menos arriscada, comparativamente às formadas por ativos das empresas dos Níveis 1 e 2 .

Como pode ser visto, essa não é uma discussão inédita para a economia brasileira. No entanto, diferencia-se das apresentadas anteriormente em alguns pontos, entre os quais, destacam-se: procura-se tratar a relação risco e retorno na forma condicional, como documentado na literatura; essa formulação se mostra adequada na explicação da variabilidade do risco esperado do portfólio em decorrência da variabilidade do risco de mercado esperado e da variância condicional do retorno de mercado; foi utilizada a formalização GARCH-M para a modelagem do risco condicional, esse modelo assume que o prêmio de risco é uma função crescente da variância condicional, ou seja, quanto maior a volatilidade condicional da série de retornos maior a compensação necessária para induzir os agentes a tomarem tal risco. 
Por fim, destaca-se que a motivação para utilizar o CAPM condicional tem pelo menos duas fontes. Do ponto de vista teórico, destaca-se que os estudos desenvolvidos por Dybvig e Ross (1985), Hansen e Richard (1987), Ferson, Kandel e Stambaugh (1987), Ferson e Harvey (1991), Wang e Jagannathan (1996) e Wang (2003) documentaram evidências de que os retornos esperados variam ao longo do tempo com a informação. Em particular, a hipótese do risco variando no tempo aparece como hipótese alternativa à explicação da realidade observada, nomeadamente por meio do CAPM condicional, uma generalização do CAPM de Sharpe, Lintner e Mossin.

Em segundo lugar, destaca-se a perspectiva empírica, visto que a literatura econométrica, de modelos de volatilidade financeira (ANDERSEN; BOLLERSLEV; DIEBOLD, 2005) proporcionou provas de grandes flutuações e persistência de alta nas variâncias condicionais de ativos financeiros. Assim, mesmo a partir de um ponto de vista meramente estatístico, betas de mercado, que são relações de variáveis no tempo, covariâncias condicionais e desvios podem ser usados para mostrar persistentes flutuações no mercado financeiro, como destacado em Bollerslev, Engle e Wooldridge (1988).

Além desta introdução, o artigo está dividido em mais cinco seções. A segunda seção faz uma abordagem da Teoria do Modelo de Precificação de Ativos de Capital (CAPM) condicional e dos princípios da governança corporativa. $\mathrm{Na}$ terceira seção são descritos os procedimentos metodológicos, sendo eles: processo de formação de carteiras, modelo de volatilidade GARCH-M e apresentação dos critérios de seleção dos dados. A quarta seção é dedicada à análise dos resultados e discussões dos testes estatísticos, sendo apresentadas as estimativas obtidas para os betas e a volatilidade condicional. A quinta seção apresenta as considerações finais, a sexta seção destina-se às referências e, por fim, são apresentados os apêndices.

\section{CAPM CONDICIONAL}

O CAPM, desenvolvido por Sharpe (1964), Lintner (1965, 1969) e Mossin (1966), marca o nascimento da teoria de precificação de ativos. Segundo Copeland, Weston e Shastri (2005), esse é um modelo de equilíbrio de mercado que possibilita a mensuração da parcela relevante do risco de um ativo individual e do prêmio pelo risco em determinado mercado. 
A importância da análise do CAPM está ligada ao processo de avaliação de tomada de decisões em condições de risco. Esse modelo mostra que existe uma relação linear entre o risco e o retorno de uma ação e o retorno do mercado acionário, em que os investidores sempre buscarão formar uma carteira que tenha a melhor relação entre o risco e o retorno, já que esses são indivíduos que procuram maximizar a utilidade esperada da sua riqueza ao longo do tempo.

Atualmente a investigação sobre o CAPM está voltada para o modelo condicional, que documenta evidências sobre a Variabilidade dos Prêmios de Risco ao Longo do Tempo (Time-Varying Risk Premium). Parte dessa evidência consiste na repetida rejeição da hipótese dos prêmios de risco esperados iguais para diferentes classes de títulos. A outra parte consiste na rejeição da hipótese dos prêmios de risco esperados e nas diferentes classes de títulos se manterem constantes ao longo do tempo.

Com essas evidências, esta pesquisa baseou-se na versão condicional do modelo CAPM proposto por Bollerslev, Engle e Wooldridge (1988). Esses autores assumem como um vetor de retornos excedentes, representado pela diferença entre o retorno nominal dos ativos e o ativo livre de risco durante o período, e assumem e como o vetor da média condicional e a matriz covariância condicional desses retornos dada a partir do conjunto de informações disponíveis no momento, assumindo ser o vetor de pesos do período, de modo que o excesso de retorno do mercado é definido como . Em seguida, o vetor de covariâncias do mercado é e o CAPM requer

$$
\mu_{\mathrm{t}}=\delta H_{\mathrm{t}} \omega_{\mathrm{t}-1}
$$

onde é uma constante de proporcionalidade, derivada por Jensen (1972), e representa uma medida agregada de aversão ao risco relativo.

A variância condicional do excesso de retorno do mercado é $\sigma_{M_{t}}^{2}=\omega_{t-1}^{\prime} H_{t} \omega_{t-1}$ e a média condicional $\mu_{M_{t}}=\omega_{t-1} \mu_{t}$, que a partir de (1) pode ser escrita como

$$
\mu_{M_{\mathrm{t}}}=\delta \sigma_{M_{\mathrm{t}}}^{2}
$$

mostrando que expressa o trade-off entre a média e a variância de mercado. Definindo o beta do ativo como a razão entre a covariância do ativo com o mercado e a variância da carteira de mercado, $\beta_{t}=H_{t} \omega_{t-1} / \sigma_{M_{t}}^{2}$ e substituindo (1) em (2), obtém-se 


$$
\mu_{\mathrm{t}}=\beta_{\mathrm{t}} \mu_{M_{\mathrm{t}}}
$$

A expressão (3) representa o modelo CAPM em termos dos momentos condicionais e reflete as informações disponíveis aos agentes no momento da tomada de decisão sobre a formação do portfólio. Destaca-se que, como a matriz de covariância dos retornos varia ao longo do tempo, os retornos médios e os betas também são variáveis com o tempo.

Em relação aos trabalhos aplicados à economia brasileira e que tratam da versão condicional do CAPM, destaca-se o trabalho desenvolvido por Bonomo e Garcia (2002), no qual destacam importantes estudos do CAPM condicional, entre eles, o proposto por Bodurtha e Mark (1991), em que o beta da carteira de ativos é definido como a covariância condicional do erro de previsão do retorno da carteira e o erro de previsão do retorno do mercado.

Já Tambosi Filho, Garcia e Bertucci (2007) procuraram testar a versão estática e dinâmica do CAPM utilizando os retornos das ações do Bovespa, da Bolsa de Valores da Argentina e da NYSE, para o Brasil, Argentina e Estados Unidos, respectivamente, entre janeiro de 1994 e dezembro de 2002. Os resultados obtidos por esses autores mostraram que, ao se compararem as estimativas do CAPM estático e condicional, o primeiro não representa satisfatoriamente a realidade econômica dos países analisados, diferentemente do CAPM condicional, que consegue captar o efeito dinâmico apresentado pelas economias, considerando os efeitos do ciclo de negócios sobre os retornos das carteiras.

Em discussão recente, Bergmann et al. (2014) analisam o retorno e o risco sistemático das carteiras de 11 setores da economia brasileira por meio do modelo do CAPM condicional não paramétrico. As ações participantes estão listadas na Bovespa no período de janeiro de 2002 a dezembro de 2009. Esses destacam que as expectativas dos retornos dos ativos se aproximam de variáveis aleatórias, já que as distribuições dos retornos dos ativos e as expectativas dos investidores se alteram de um período para outro. Assim, o modelo do CAPM condicional se sobressai ao modelo do CAPM tradicional, que assume que as variáveis são constantes, fazendo com que, nesse caso, as estimativas obtidas a partir desse modelo não sejam realistas.

\subsection{GOVERNANÇA CORPORATIVA}

Esta seção foi desenvolvida com base nos princípios de governança corporativa proposta pela Bolsa de Valores, Mercadorias e Futuros (BM\&FBovespa), 
segundo a qual a premissa básica dos segmentos especiais de listagem é que a melhoria da qualidade das informações prestadas pela companhia e a ampliação dos direitos societários reduzem as incertezas no processo de avaliação e de investimento e, consequentemente, o risco. Assim, eleva-se a disposição dos investidores de adquirir ações da companhia, tornando-se sócios. A redução do risco também gera melhor precificação das ações, o que, por sua vez, incentiva novas aberturas de capital e novas emissões, fortalecendo o mercado acionário como alternativa de financiamento às empresas.

Dessa forma, o Novo Mercado e os Níveis Diferenciados de Governança Corporativa - Nível 1 e Nível 2 - são segmentos especiais de listagem desenvolvidos com o objetivo de proporcionar um ambiente de negociação que estimulasse, ao mesmo tempo, o interesse dos investidores e a valorização das companhias.

A maior parte dos compromissos que as empresas do Nível 1 assumem perante os investidores se refere ao fornecimento de informações que auxiliam na avaliação sobre o valor da empresa. Além disso, a empresa Nível 1 não deve ter partes beneficiárias.

A principal diferença entre o Novo Mercado e o Nível 2 se refere à emissão de ações preferenciais. Enquanto as empresas do Novo Mercado somente possuem ações ordinárias (todos têm direito de voto), as empresas do Nível 2 têm ações preferenciais, muito embora os titulares dessas ações preferenciais tenham direito de voto em algumas matérias específicas.

De acordo com as informações apresentadas pela BM\&FBovespa, o Novo Mercado representa um novo segmento de compromissos de governança corporativa, em que tais compromissos se referem à prestação de informações que facilitam o acompanhamento e a fiscalização dos atos da administração e dos controladores da companhia, e à adoção de regras societárias que melhor equilibram os direitos de todos os acionistas, independentemente da sua condição de controlador ou investidor.

A melhoria da qualidade das informações prestadas pela companhia e a ampliação dos direitos societários reduzem as incertezas no processo de avaliação e de investimento e, consequentemente, o risco. Assim, em virtude do aumento da confiança, eleva-se a disposição dos investidores de adquirir ações da companhia, tornando-se sócios desta. A redução do risco também gera melhor precificação das ações, o que, por sua vez, incentiva novas aberturas de capital e novas emissões, fortalecendo o mercado acionário como alternativa de financiamento às empresas. 


\section{PROCEDIMENTOS METODOLÓGICOS}

\subsection{FORMAÇÕES DAS CARTEIRAS}

O processo de análise e seleção de carteiras nesta pesquisa está baseado no trabalho desenvolvido por Markowitz (1952). Uma hipótese importante da teoria do portfólio (carteira) é que o risco de um ativo mantido fora de uma carteira é diferente de seu risco quando incluído na carteira. Se o mercado for eficiente, então todos os portfólios diversificados são preferíveis a todos os portfólios não diversificados.

Assim, o retorno da carteira será representado por:

$$
E\left(R_{p}\right)=\sum_{i=1}^{n} R_{i} X_{i}
$$

onde $X_{i}$ representa a participação da ação, sendo $\sum X_{i}=1$ e $R_{i}$ o retorno da ação $i$.

E o risco do portfólio $\sigma_{p}$ será obtido a partir da seguinte expressão:

$$
\sigma_{p}=\left[\sum_{i=1}^{n} \sum_{j=1}^{n} X_{i} X_{j} \rho_{i j} \sigma_{i} \sigma_{j}\right]^{\frac{1}{2}}
$$

onde $X_{i}$ e $X_{j}$ representa a participação das ações $i$ e $j$ na carteira; $\rho_{i j}$ é o coeficiente de correlação entre os ativos; $\sigma_{i}$ e $\sigma_{j}$ é o desvio padrão de cada ativo.

Para otimizar as carteiras de ações será utilizada a ferramenta solver do Excel, sendo adotadas as seguintes restrições: as participações das ações nas carteiras serão não negativas, a soma das participações não pode exceder $100 \%$ e a taxa de retorno mínima desejada será $0,03 \%$, obtida a partir do retorno médio do ativo livre de risco (CDI).

\subsection{MODELO DE VOLATILIDADE}

A modelagem da volatilidade em séries temporais financeiras tem sido objeto de atenção desde a introdução dos modelos autorregressivos com heterocedasticidade 
condicional (ARCH), no paper seminal de Engle (1982). Os modelos ARCH se caracterizam por apresentar retorno não correlacionado serialmente e a volatilidade depende de retornos passados por meio de uma função quadrática. Numerosas variantes e extensões dos modelos ARCH têm sido propostas nas últimas décadas.

O modelo ARCH-M, ou ARCH em média, proposto por Engle, Lilien e Robins (1987) representa uma das extensões do modelo ARCH. No modelo ARCH-M, a média condicional é uma função explícita da variância condicional, como determinado em (6), assim:

$$
\mu_{i t}=\alpha_{i}+\beta_{i}\left(r_{m t}-r_{f t}\right)+\delta_{i} h_{t}
$$

onde $\delta$ representa o prêmio de risco; $\mu_{i t}$ corresponde à média condicional dos retornos e $h_{t}$ representa a volatilidade condicional.

Nesse modelo, o aumento da variância condicional está associado ao aumento ou diminuição da média de condicional $\left(\mu_{i t}\right)$. Para completar a especificação do modelo $\left(h_{t}\right)$, a variância condicional deve ser parametrizada em razão do conjunto de informações passadas.

$$
h_{t}=\alpha_{0}+\sum_{i=1}^{q} \alpha_{i} r_{t-i}^{2}
$$

onde $\alpha_{i}$ representa a presença do efeito ARCH.

Desse modo, esse modelo permite que a variância condicional possa afetar a média condicional. Em outras palavras, alterações na variância condicional afetam diretamente o retorno esperado das ações.

O modelo GARCH-M representa a generalização do modelo ARCH-M. Os modelos GARCH-M assumem que o prêmio de risco é uma função crescente da variância condicional, ou seja, quanto maior a volatilidade condicional da série de retornos maior a compensação necessária para induzir os agentes a tomarem tal risco. O processo GARCH-M pode ser descrito como:

$$
\mu_{i t}=a_{i}+b_{i}\left(r_{m t}-r_{f t}\right)+\delta_{i} h_{t} \delta>0
$$




$$
h_{t}=\alpha_{0}+\sum_{i=1}^{p} \alpha_{i} r_{t-i}^{2}+\sum_{j=1}^{q} \beta_{j} h_{t-j}
$$

onde $\delta$ representa o prêmio pelo risco. Quando o prêmio pelo risco é positivo indica que a média condicional do retorno é positivamente relacionada com a variância condicional passada.

O modelo GARCH-M também permite quantificar a influência da persistência da volatilidade nos retornos por meio do cálculo da meia-vida, que mede o período de tempo necessário para que o efeito de choque na volatilidade diminua até a metade, e é dado por $\tau=1-[\log 2 / \log \gamma]$, onde $\gamma=(\alpha+\beta)$.

\subsection{DADOS}

Com o objetivo de mensurar o risco associado às empresas que apresentam níveis diferenciados de governança corporativa, foram coletados dados referentes aos preços de fechamento diários das empresas vinculadas aos seus respectivos níveis de governança. Salienta-se que para proceder à estimativa dos betas das carteiras de investimento foi necessária a obtenção dos retornos das séries temporais financeiras, do ativo livre de risco (CDI) e da carteira de mercado (IBovespa).

O período de análise se estende de 04 de janeiro de 2010 a 17 de janeiro de 2014, com os retornos diários sendo calculados em base contínua. Como descrito anteriormente, a escolha do período de análise está em conformidade com a disponibilidade dos dados para as ações classificadas no Nível de governança N2. Nesse caso, a ampliação da janela temporal restringiria o número de ações presentes nessa carteira. Na definição da amostra, foram selecionadas 37 empresas para o Nível de governança N1, 14 para o N2 e 67 para o NM, sendo eliminadas 76 empresas por não apresentarem periodicidade completa para o período analisado.

\section{RESULTADOS}

Nesta seção serão apresentados os resultados obtidos pelo processo de otimização de carteira para os níveis de governança corporativa N1, N2 e NM, as estimativas dos betas variando no tempo, obtidas a partir do CAPM condicional e, por fim, a análise de volatilidade condicional de níveis diferenciados de governança 
corporativa. O objetivo é entender se níveis diferenciados de governança corporativa afetam os riscos de investimento em carteira.

\subsection{ANÁLISE RISCO VERSUS RETORNO}

A discussão subsequente irá apresentar os resultados obtidos pelo processo de otimização de carteira para os Níveis de governança corporativa N1, N2 e NM. O Gráfico 1 mostra a relação entre os excessos dos retornos das carteiras com níveis diferenciados de governança corporativa e o excesso de retorno da carteira de mercado.

Ao se analisarem os gráficos de dispersão entre essas séries de excessos dos retornos das carteiras com níveis diferenciados de governança corporativa e o excesso de retorno da carteira de mercado não fica clara a presença de uma associação linear entre esses termos. No entanto, ao se analisar o desvio padrão entre essas séries é possível verificar que a carteira N1 possui dispersão maior que as demais carteiras.

Gráfico 1 - Relação entre os excessos dos retornos das carteiras com níveis diferenciados de governança corporativa e o excesso de retorno da carteira de mercado
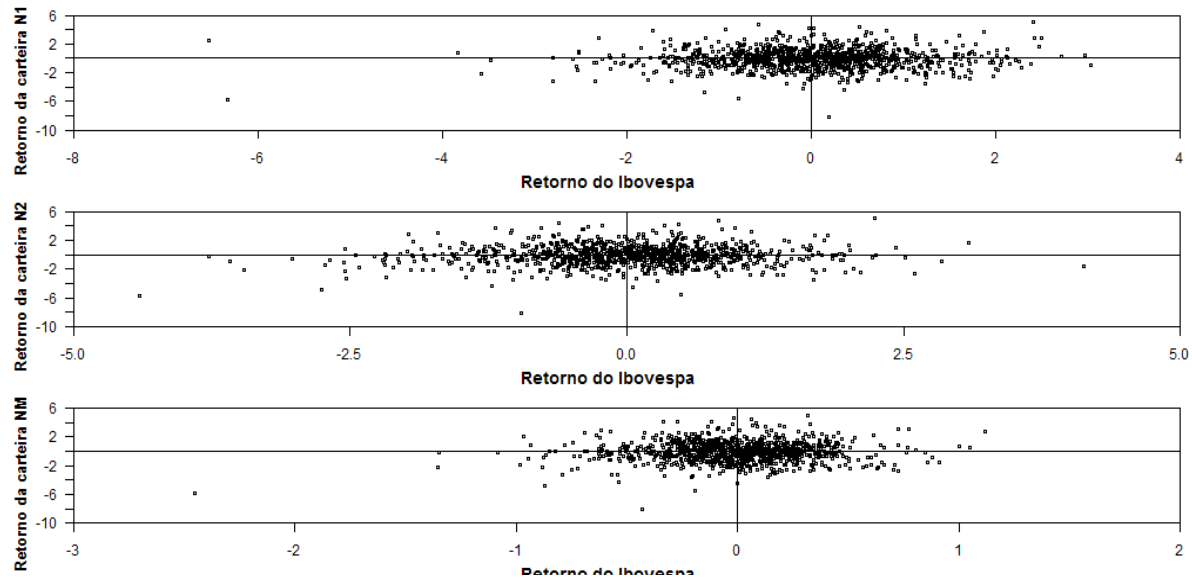

Fonte: os autores.

Outra ferramenta que pode ser adotada na investigação da relação entre o risco e o retorno das carteiras formadas e a carteira de mercado é dada pela avaliação de tomada de decisões em condições de risco. Para esse fim será utilizado o Capital Asset Pricing Model (CAPM), desenvolvido por Sharpe (1964), Lintner (1965, 1969) e Mossin (1966). Ao se proceder a essa análise tradicional é possível ter uma noção 
mais precisa da relação entre o excesso de retorno das carteiras analisadas e o excesso de retorno da carteira de mercado.

As estimativas dos coeficientes do CAPM estático são descritas na Tabela 1, sendo possível verificar que a carteira de Novo Mercado apresentou um baixo nível de risco associado às suas ações em relação às carteiras de Nível 1 e Nível 2.

Tabela 1 - Estimativas dos betas médios obtidos por meio do modelo CAPM estático

\begin{tabular}{cr}
\hline Carteiras & (Mensal) \\
\hline Nível 1 & 0,5 \\
Nível 2 & 0,816 \\
Novo Mercado & 0,242 \\
\hline
\end{tabular}

Fonte: os autores.

Com isso, a primeira impressão que surge é a de que carteiras formadas a partir de ações classificadas no Novo Mercado possuem risco inferior às carteiras formadas a partir dos outros níveis de governança. No entanto, surgem alguns questionamentos importantes na descrição dessas estimativas: Será que a relação risco e retorno descrita na Tabela 1 é verdadeira? Essas estimativas se mantêm fixas ao longo do tempo? Ou momentos de instabilidade econômica possuem o mesmo peso na explicação dessa relação que os momentos de estabilidade? Em relação aos dois últimos questionamentos, provavelmente, a resposta é negativa.

Essa conclusão é documentada por estudos como os de Dybvig e Ross (1985), Hansen e Richard (1987), Ferson, Kandel e Stambaugh (1987), Ferson e Harvey (1991), Wang e Jagannathan (1996) e Wang (2003), que mostraram que as condições econômicofinanceiras sugerem betas que devem variar de acordo com variáveis condicionais, uma ideia desenvolvida teórica e empiricamente. Dada essa regularidade teórica e empírica, impõe-se uma condição de investigação importante na nossa análise, que é a discussão dos betas variando ao longo do tempo. A não incorporação das mudanças de cenários econômicos e os possíveis problemas teóricos apresentados pela versão estática do modelo CAPM levam ao uso da versão condicional do CAPM. A Tabela 2 descreve as características dos betas estimados por meio do modelo CAPM condicional.

Tabela 2 - Análise estatística dos betas condicionais das carteiras de Nível N1, N2 e NM

\begin{tabular}{lrrr}
\hline \multicolumn{1}{c}{ Descrição } & Beta N1 & Beta N2 & \multicolumn{1}{c}{ Beta NM } \\
\hline Média & 0.026 & 0.012 & 0.007 \\
Desvio padrão & 0.049 & 0.080 & 0.028 \\
Mínimo & -0.081 & -0.088 & -0.050 \\
Máximo & 0.123 & 0.246 & 0.124 \\
\hline
\end{tabular}

Fonte: os autores. 
É possível observar, a partir da Tabela 2, que a carteira de Nível N1 apresentou retorno médio superior às demais carteiras. Em outras palavras, o aumento de $1 \%$ no retorno da carteira de mercado representa um crescimento de 2,6, 1,2 e 0,7\%, respectivamente, nos retornos das carteiras citadas anteriormente. Dessa forma, para o período analisado, as carteiras de Nível 1 e Nível 2 se mostraram agressivas em relação à carteira de mercado, diferentemente da carteira de Novo Mercado, que apresentou perfil conservador em relação à carteira de mercado.

Por outro lado, o fato de as carteiras de Nível 1 e Nível 2 apresentarem retorno maior que o retorno da carteira de Novo Mercado também representa que o risco associado a essas ações é maior que o risco associado à carteira de Novo Mercado. As estimativas dos betas variando ao longo do tempo são expressas no Gráfico 2.

Gráfico 2 - Betas condicionais das carteiras de Nível N1, N2 e NM
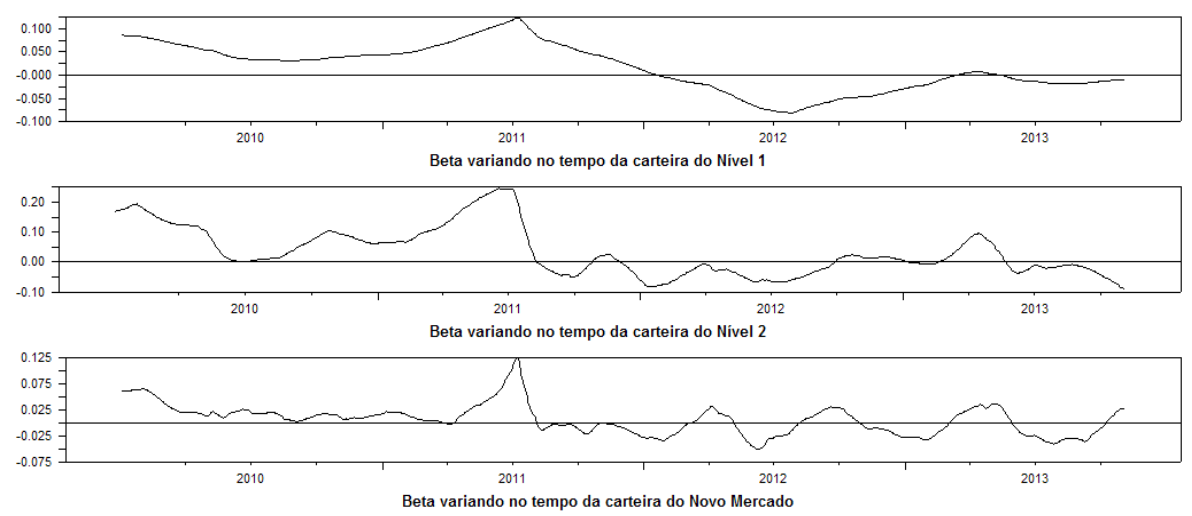

Fonte: os autores.

Ao se proceder à análise por meio do CAPM condicional é possível segmentar algumas discussões suprimidas pelas estimativas obtidas pelo beta médio. Uma dessas conclusões é que os betas apresentaram valores negativos para alguns períodos, indicando que os retornos das carteiras criadas mudaram no sentido contrário ao retorno da carteira de mercado. Em relação ao objetivo da pesquisa, percebe-se a partir do Grafico 2 que o intervalo de variação dos betas da carteira de Novo Mercado é inferior ao intervalo das outras carteiras, mostrando que o risco dessa carteira é inferior aos das demais. 


\subsection{ESTIMATIVAS DA VOLATILIDADE}

Para o desenvolvimento da análise de volatilidade foram plotadas, inicialmente, as séries de retornos das carteiras de variância mínima composta para os três níveis de governança estudados. Na sequência serão abordadas as características estatísticas das séries de retorno e, por fim, apresentadas as estimativas das volatilidades ou risco das carteiras.

Gráfico 3 - Séries de excesso de retorno das carteiras de Nível N1, N2 e NM



Fonte: os autores.

A partir do Gráfico 3 é possível destacar algumas características importantes, como:

a) aglomeração de volatilidade nas séries de retorno;

b) a série de retornos da carteira de Novo Mercado apresentou volatilidade inferior às demais carteiras;

c) existência de períodos de alta volatilidade, representando um indicativo de autocorrelação entre os retornos.

É importante salientar que a descrição adotada para a representação da distribuição conjunta dos retornos adotada nesta pesquisa está baseada na taxa de crescimento entre o conjunto de informação no instante $t$ em relação aos acontecimentos de instantes anteriores.

Do ponto de vista estatístico, as séries de retornos se caracterizam por apresentar caudas mais pesadas que a distribuição normal, implicando problemas de normalidade. A Tabela 3 apresenta algumas estatísticas descritivas das séries de retornos das carteiras analisadas e, como 
pode ser visto, todas as carteiras rejeitaram a hipótese de normalidade. O teste de Jarque-Bera e seus respectivos p-valores indicam a rejeição da hipótese nula ao nível de confiança de 95\%.

Tabela 3 - Estatísticas descritivas das séries de retornos das carteiras N1, N2 e NM

\begin{tabular}{lrrr}
\hline \multicolumn{1}{c}{ Estatística } & Carteira N1 & Carteira N2 & Carteira NM \\
\hline Média & -0.001 & -0.003 & -0.015 \\
Erro padrão & 0.96 & 0.91 & 0.31 \\
Assimetria & -0.69 & -0.36 & -0.64 \\
Curtose & 4.13 & 1.78 & 4.38 \\
Jarque-Bera & 792.39 & 155.22 & 874.04 \\
Probabilidade & 0.00 & 0.00 & 0.00 \\
\hline
\end{tabular}

Fonte: os autores.

${ }^{\star}$ Nota: O teste de Jarque-Bera rejeita a hipótese de normalidade das séries de retornos.

Analisando o terceiro e o quarto momentos da distribuição dos retornos dos portfólios representados, respectivamente, pela medida de curtose e assimetria, é possível verificar que as séries de retorno possuem assimetria e excesso de curtose na distribuição. Em relação ao excesso de curtose, esse indicador pode ser interpretado como distribuição com "cauda pesada" e com grande probabilidade de ocorrerem valores longe da média em uma distribuição. Por outro lado, a assimetria mostra quão assimétrica é a curva de distribuição em relação à média; na nossa investigação foi possível constatar que, entre as distribuições de retornos, a carteira N2 apresentou maior assimetria negativa.

Ao se comparar a distribuição dos retornos com as distribuições $t$-Student e normal, é possível verificar que a distribuição t-Student apresenta performance melhor para expressar as séries de retornos.

Gráfico 4 - Análise comparativa entre as distribuições dos retornos com as distribuições $t$-Student e normal

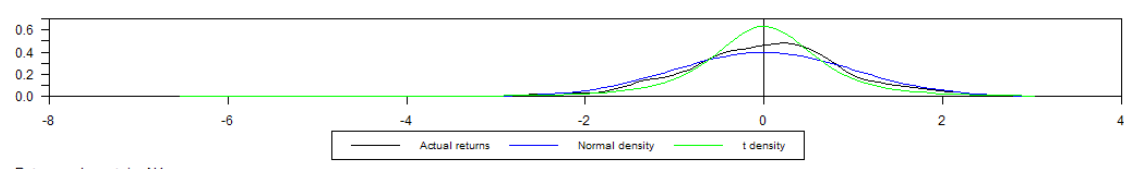

Retornos da carteira N1

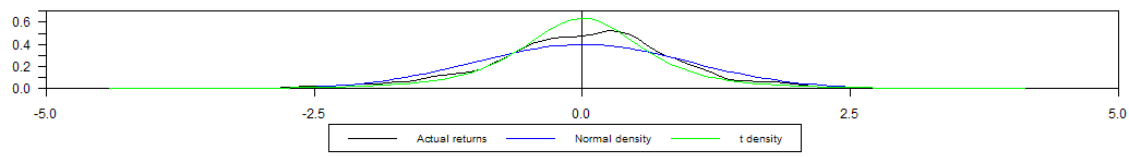

Retornos da carteira N2

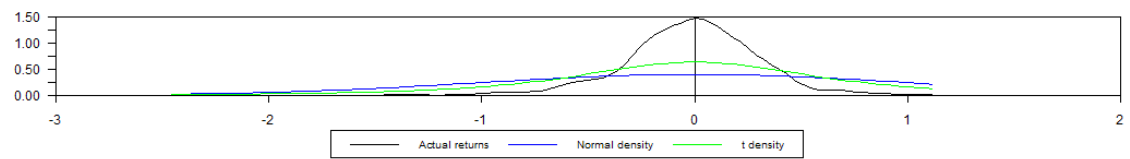

Fonte: os autores. 
Não parece razoável corrigir essas distorções em relação à distribuição normal, mas tendo conhecimento dessas características é importante adotar uma modelagem de volatilidade que incorpore as características típicas de séries financeiras em suas estruturas, como acomodação de caudas pesadas e distribuições assimétricas. Dessa forma, o objetivo da modelagem subsequente é a estimação da variância das distribuições condicionais das séries de retornos, também denominada volatilidade por meio do modelo GARCH-M. A adoção de uma especificação adequada para a modelagem da volatilidade das séries de retornos exige a realização de alguns testes estatísticos iniciais, como ARCH-LM e o uso de critérios AIC e SBC para o processo de seleção de modelos.

A importância de adotar critérios de seleção está ligada ao fato de que incorporar novos termos no modelo pode aumentar o grau de ajustamento deste, bem como aumentar a variância do erro de previsão. Portanto, uma forma de impor restrições sobre o acréscimo de regressores é dada pelos critérios AIC e SBC. Como pode ser visto na Tabela B.1 (Apêndice B), os modelos selecionados para estimar a volatilidade das carteiras foram o GARCH-M $(1,1)$; o processo de seleção foi determinado pelo critério de mínimo AIC e SBC.

Por fim, foi aplicado o teste de ARCH-LM para verificar a presença de heterocedasticidade condicional nos modelos descritos na Tabela B.2 (Apêndice B). Na presença de heterocedasticidade há perda de eficiência dos estimadores e a variância do termo do aleatório apresenta dependência temporal em relação a choques na volatilidade passada e, com isso, o estimador deixa de apresentar variância mínima.

A partir da Tabela B.2 foi possível identificar a ausência de heterocedasticidade condicional nos resíduos dos modelos, ou seja, com um nível de significância de 5\%, o teste de ARCH-LM aceitou a hipótese de ausência de heterocedasticidade condicional. Dessa forma é possível concluir que os estimadores são eficientes estatisticamente, fato que valida as análises que serão realizadas sobre o mercado financeiro brasileiro.

Passada a análise estatística, o enfoque passa a ser a estimativa da volatilidade. Para avaliar se a melhoria da qualidade das informações prestadas pelas companhias e a ampliação dos direitos societários reduzem risco, foi aplicada a análise da persistência na volatilidade das carteiras, por meio da estimação dos modelos da família ARCH.

A partir da Tabela 4 é possível demonstrar que a melhor estrutura para formalizar a variância condicional das empresas, com diferentes níveis de governança, é atribuída pelos modelos GARCH-M $(1,1)$. 
Tabela 4 - Equações da variância obtidas a partir do modelo GARCH-M

\begin{tabular}{lrrrr}
\hline \multicolumn{1}{c}{ Carteiras } & \multicolumn{1}{c}{ Constante } & & & \\
\hline \multirow{2}{*}{$\mathrm{N} 1$} & 0.05 & 0.11 & 0.83 & 0.94 \\
& $(0.02)$ & $(0.02)$ & $(0.03)$ & \\
$\mathrm{N} 2$ & 0.03 & 0.08 & 0.89 & \\
& $(0.01)$ & $(0.01)$ & $(0.02)$ & 0.97 \\
$\mathrm{~N}$ & 0.01 & 0.07 & 0.85 & \\
& $(0.002)$ & $(0.02)$ & $(0.03)$ & 0.92 \\
\hline
\end{tabular}

Fonte: os autores.

${ }^{\star}$ Nota: Os termos entre parênteses representam o desvio padrão.

A persistência representa as manifestações do agrupamento da volatilidade. Em outras palavras, representa o período de tempo que os efeitos de choques sofridos na variância das ações irão levar para se dissipar. Como descrito na seção 3.2, a persistência do impacto resultante de choques na variância é dada pelo somatório dos parâmetros e do modelo GARCH-M. Quanto mais próxima essa soma ficar de 1, maior será o efeito daquela informação (choque) no decorrer do tempo, levando mais dias para se dissipar.

Verificou-se na Tabela 4 que o coeficiente de persistência apresentou diferenças significativas entre os três níveis de governança. No caso específico das ações classificadas no Nível 1 e no Nível 2 de governança, o coeficiente de persistência se aproximou da unidade, indicando que a característica da volatilidade apresentada nesses níveis de governança é do tipo persistente. Logo, perdurarão por muito tempo, nos preços dessas ações, os efeitos de um choque exógeno até se dissipar por completo. De outro modo, a evidência do agrupamento da volatilidade nos retornos dessas ações significa que esse investimento é caracterizado por ser arriscado.

As ações das empresas listadas no Novo Mercado apresentaram coeficiente de persistência inferior ao obtido pelas carteiras N1 e N2, indicando que os efeitos dos choques na volatilidade dos retornos levam menos tempo para se dissipar. Assim, investir em ações que, como descrito na seção 3.2, apresentam melhoria na qualidade das informações prestadas pela companhia e a ampliação dos direitos societários reduz as incertezas no processo de avaliação e de investimento e, consequentemente, o risco.

A discussão apresentada fica mais clara ao se analisar a partir do Gráfico 5, em que são apresentadas as séries de volatilidades estimadas para as carteiras N1, N2 e NM. Uma vez que foi identificado que as séries de retornos apresentaram melhor ajuste ao modelo GARCH-M (1,1), foi possível analisar de forma individual a 
dinâmica da volatilidade das séries de retornos das carteiras com níveis diferenciados de governança corporativa.

Gráfico 5 - Variância dos retornos das carteiras de Nível N1, N2 e NM, estimadas a partir do modelo GARCH-M $(1,1)$

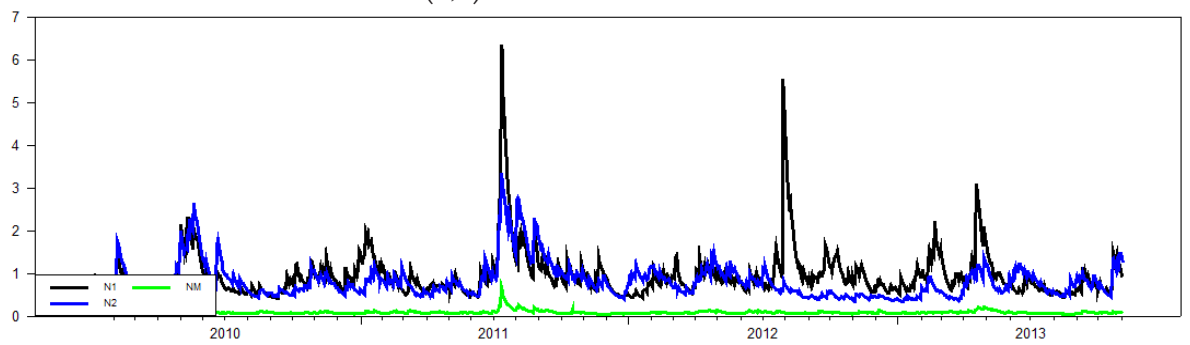

Fonte: os autores.

Inicialmente, pode-se destacar que existem evidências de que em alguns momentos a volatilidade das carteiras $\mathrm{N} 1$ e $\mathrm{N} 2$ caminham juntas, enquanto que em outros a carteira N2 apresenta movimentos mais suaves que a carteira N1. Por meio desta análise, comprova-se que as ações das empresas do Novo Mercado são as menos voláteis, seguidas pelas ações classificadas no Nível 2 e no Nível 1 (mais volátil), respectivamente. Esses resultados reforçam a hipótese de que a qualidade das informações prestadas pelas empresas e a ampliação dos direitos societários reduzem os riscos de investimento.

\section{CONSIDERAÇÕES FINAIS}

O objetivo geral deste estudo consistiu em verificar se a qualidade das informações prestadas pelas empresas e a ampliação dos direitos societários reduzem os riscos de investimento. Para tanto, utilizou-se um arcabouço teórico baseado nos desdobramentos do CAPM condicional e, empiricamente, analisou-se a validade das estimativas da volatilidade condicional utilizando os três segmentos de governança corporativa do Nível 1 (N1), Nível 2 (N2) e Novo Mercado (NM). A análise implementada neste trabalho permite destacarem-se algumas considerações importantes.

Em primeiro lugar, ao se proceder à análise por meio do CAPM condicional foi possível apresentar algumas discussões suprimidas pelas estimativas obtidas pelo beta médio. Uma dessas conclusões é que os betas apresentaram valores negativos para alguns períodos, indicando que os retornos das carteiras criadas mudaram 
no sentido contrário ao retorno da carteira de mercado. Em relação ao objetivo da pesquisa, percebe-se que o intervalo de variação dos betas da carteira de Novo Mercado é inferior aos das outras carteiras, apresentando um primeiro indício de que o risco dessa carteira é inferior aos das demais.

Verificou-se que as ações das empresas listadas no Novo Mercado apresentaram coeficiente de persistência inferior aos obtidos pelas carteiras $\mathrm{N} 1 \mathrm{e}$ $\mathrm{N} 2$, indicando que os efeitos dos choques negativos na volatilidade dos retornos levam menos tempo para se dissipar nessa carteira. E, por meio das estimativas das volatilidades, também foi possível comprovar que as ações das empresas do Novo Mercado são as menos voláteis, seguidas pelas ações classificadas no Nível 2 e no Nível 1 (mais volátil), respectivamente. Esses resultados reforçam a hipótese que a qualidade das informações prestadas pelas empresas e a ampliação dos direitos societários reduzem os riscos de investimento.

Em relação às perspectivas futuras desta pesquisa, pretende-se incluir na análise as carteiras setoriais com o propósito de simular cenários de choques negativos na economia brasileira e verificar o desempenho da volatilidade dessas carteiras em relação ao comportamento das carteiras com diferentes níveis de governança corporativa.

\section{REFERÊNCIAS}

ANDERSEN, T. G.; BOLLERSLEV, T.; DIEBOLD, F. X. Parametric and nonparametric volatility measurement. In: HANSEN, L. P.; AIT-SAHALIA, Y. (Ed.). Handbook of financial econometrics. Amsterdam: North-Holland, 2005.

BATISTELLA, F. D. et al. Retornos de ações e governança corporativa: um estudo de eventos. In: CONGRESSO USP DE CONTROLADORIA E CONTABILIDADE, 1., 2004, São Paulo. Anais... São Paulo: USP, 2004.

BERGMANN, D. R. et al. Testing the Non-Paramentric Conditional CAPM in the Brazilian Stock Market. Revista de Ciências da Administração, v. 16, n. 38, p. 213-227, abr. 2014.

BODURTHA JUNIOR, J. N.; MARK, Nelson C. Testing the CAPM with time varying risks and returns. Journal of Finance, v. 46, p. 1485-1505, 1991. 
BOLlERSLEV, T.; ENGLE, R. F.; WOOLDRIDGE, J. M. A Capital Asset Pricing Model with Time-varying Covariances. Journal of Political Economy, v. 96, n. 1, Feb. 1988.

BONOMO, M.; GARCIA, R. Estimando e testando o CAPM condicional com efeitos ARCH para o mercado acionário brasileiro. In: BONOMO, M. (Ed.).

Finanças aplicadas ao Brasil. Rio de Janeiro: Ed. FGV, 2002.

BORGES, B. K.; CALDEIRA, J. F.; ZIEGELMANN, F. A. Seleção de Carteira de Variância Mínima Usando dados intradiários: uma comparação empírica entre diferentes medidas realizadas para dados da BM\&FBovespa. Porto Alegre: Universidade Federal do Rio Grande do Sul, 2013.

CARVALHO, A. G. de. Governança corporativa no Brasil em perspectiva. Revista de Administração da Universidade de São Paulo, v. 37, n. 3, 2002.

CLEMENTE, A.; MATUCHESKI, S.; SANDRINI, J. C. Governança corporativa e volatilidade das ações negociadas na Bovespa na crise financeira de 2008.

REBRAE - Revista Brasileira de Estratégia, Curitiba, v. 2, n. 2, p. 171-183, maio/ago. 2009.

COPELAND, T. E.; WESTON, J. F.; SHASTRI, K. Financial theory and corporate policy. USA: Pearson Addison Wesley, 2005.

DYBVIG, P. H.; ROSS, S. A. Differential Information and Performance Measurement Using a Security Market Line. The Journal of Finance, v. 40, n. 2, p. 383-399, June 1985.

ENGLE, R. F. Autoregressive Conditional Heteroscedasticity with Estimates of the Variance of United Kingdom Inflation. Econometrica, v. 50, n. 4, July 1982.

ENGLE, R. F.; LILIEN, D. M.; ROBINS, R. P. Estimating Time Varying Risk Premia in the Term Structure: the ARCH-M Model. Econometrica, v. 55, n. 2, p. 391-407, Mar. 1987.

FERSON, W. E.; HARVEY, C. R. The Variation of Economic Risk Premiums. Journal of Political Economy, v. 99, n. 2, Apr. 1991.

FERSON, W. E.; KANDEL, S.; STAMBAUGH, R. F. Tests of Asset Pricing with Time-Varying Expected Risk Premiums and Market Betas. The Journal of Finance, v. 42, n. 2, p. 201-220, June 1987.

FIRMINO, R. G. et al. A Relação dos Níveis de Governança Corporativa e Índices de Rentabilidade Econômica. Belo Horizonte: REUNA, 2012. 
HANSEN, L. P.; RICHARD, S. F. The Role of Conditioning Information in Deducing Testable Restrictions Implied by Dynamic Asset Pricing Models.

Econometrica, v. 55, n. 3, p. 587-613, May 1987.

JENSEN, M. C. The Foundations and Current State of Capital Market Theory. The Bell Journal of Economics and Management Science, v. 3, n. 2, 1972.

KALATZIS, A. E. G.; SIRQUEIRA, A. B. de; TOLEDO, F. M. B. Boas Práticas de Governança Corporativa e Otimização de Portfólio: uma análise comparativa. In: ENCONTRO ANPAD, 32., 2008, Rio de Janeiro. Anais... Rio de Janeiro: ANPAD, 2008.

LINTNER, J. The aggregation of investors diverse judgments and preferences in purely competitive security markets. Journal of Financial and Quantitative Analysis, v. 4, p. 347-400, 1969.

LINTNER, J. The Valuation of Risk Assets and the Selection of Risky Investments in Stock Portfolios and Capital Budgets. The Review of Economics and Statistics, v. 47, Feb. 1965.

MALACRIDA, M. J. C.; YAMAMOTO, M. M. Governança corporativa: nível de evidenciação das informações e sua relação com a volatilidade das ações do Ibovespa. RC\&F - Revista de Contabilidade e Finanças da USP, São Paulo, p. 65-79, 2006. Edição Comemorativa.

MARKOWITZ, H. Portfolio Selection. The Journal of Finance, v. 7, n. 1, p. 77-91, Mar. 1952.

MARTINS, S. M. et al. Governança corporativa: teoria e prática. Revista Eletrônica de Gestão de Negócios, v. 1, n. 3, p. 76-90, 2005.

MONTE, P. A. do et al. Existe relação entre Governança Corporativa e volatilidade? Um estudo a partir da formação de carteiras. Contabilidade Vista \& Revista, v. 21, n. 2, p. 15-44, 2010.

MOSSIN, J. Equilibrium in a capital asset market. Econometrica, v. 34, n. 4, Oct. 1966.

OLIVEIRA, L. S. S. de; SANTOS, D. F. L. Desempenho e Volatilidade dos Índices de Governança Corporativa da BM\&FBOVESPA. REUNIR - Revista de Administração, Contabilidade e Sustentabilidade, v. 1, n. 2, p. 52-64, set./ dez. 2011. 
ROGERS, P.; MACHADO FILHO, C. A. P.; SECURATO, J. R. Governança corporativa, risco operacional e comportamento e estrutura a termo da volatilidade no mercado de capitais brasileiro. In: ENCONTRO NACIONAL DE PESQUISA EM ADMINISTRAÇÃO, 32., 2008, Rio de Janeiro. Anais... Rio de Janeiro: ANPAD, 2008.

SHARPE, W. F. Capital Asset Prices: A Theory of Market Equilibrium under Conditions of Risk. The Journal of Finance, v. 19, n. 3, p. 425-442, Sept. 1964.

TAMBOSI FILHO, E.; GARCIA, F. G.; BERTUCCI, L. A. Testando

Empiricamente o CAPM condicional dos retornos esperados de carteiras dos mercados brasileiro, argentino e norte-americano. Revista de Gestão USP, v. 14, n. 4, p. 63-75, out./dez. 2007.

VENTURA, A. F. A. et al. A Relação entre os Níveis de Governança Corporativa e a Rentabilidade Econômica. REUNA, v. 17, n. 3, p. 73-84, 2012.

WANG, K. Q. Asset pricing with conditioning information: A new test. Journal of Finance, v. 58, p. 161-96, 2003.

WANG, Z.; JAGANNATHAN, R. The Conditional CAPM and the CrossSection of Expected Returns. The Journal of Finance, v. 51, n. 1, p. 3-53, Mar. 1996. 


\section{APÊNDICE A - COMPOSIÇÃO DAS CARTEIRAS N1, N2 E NM}

Tabela A.1 - Retorno, risco e participação das ações utilizadas na formação das carteiras N1, N2 e NM

(continua)

\begin{tabular}{|c|c|c|c|c|}
\hline Nome da ação & $\begin{array}{c}\text { Nível de } \\
\text { Gover- } \\
\text { nança }\end{array}$ & Retorno esperado & $\begin{array}{c}\text { Risco } \\
\text { Esperado }\end{array}$ & Participação \\
\hline BRSR6 & N1 & $0,00056088 \%$ & $0,00066950 \%$ & $3,99577289 \%$ \\
\hline BICB4 & N1 & $0,00005682 \%$ & $0,00006317 \%$ & $0,50176872 \%$ \\
\hline BBDC3 & N1 & $0,00021006 \%$ & $0,00024888 \%$ & $1,55045397 \%$ \\
\hline BBDC4 & N1 & $0,00042151 \%$ & $0,00050423 \%$ & $2,95566112 \%$ \\
\hline BRAP3 & N1 & $0,00026678 \%$ & $0,00031877 \%$ & $1,86352514 \%$ \\
\hline BRAP4 & N1 & $0,00145694 \%$ & $0,00181058 \%$ & $8,64670580 \%$ \\
\hline BRKM3 & N1 & $0,00013723 \%$ & $0,00015065 \%$ & $1,20617271 \%$ \\
\hline BRKM5 & N1 & $0,00091492 \%$ & $0,00114003 \%$ & $5,51094935 \%$ \\
\hline CMIG3 & N1 & $0,00003091 \%$ & $0,00003002 \%$ & $0,34644230 \%$ \\
\hline CMIG4 & N1 & 0,00011999\% & $0,00012154 \%$ & $1,18787266 \%$ \\
\hline CESP6 & N1 & $0,00045338 \%$ & $0,00048764 \%$ & $3,87607186 \%$ \\
\hline CPLE3 & N1 & $0,00000505 \%$ & $0,00000471 \%$ & $0,06167223 \%$ \\
\hline CPLE6 & N1 & $0,00007834 \%$ & $0,00008064 \%$ & 0,79291381\% \\
\hline ELET3 & N1 & $0,00099453 \%$ & $0,00118949 \%$ & $6,83835861 \%$ \\
\hline ELET6 & N1 & $0,00032216 \%$ & $0,00037747 \%$ & $2,48094095 \%$ \\
\hline EUCA4 & N1 & $0,00014404 \%$ & $0,00015613 \%$ & $1,29557363 \%$ \\
\hline FESA4 & N1 & $0,00040845 \%$ & $0,00048285 \%$ & $2,94091775 \%$ \\
\hline GGBR3 & N1 & $0,00068132 \%$ & $0,00086264 \%$ & $3,84551754 \%$ \\
\hline GGBR4 & N1 & $0,00131963 \%$ & $0,00168618 \%$ & $7,07781779 \%$ \\
\hline GOAU4 & N1 & $0,00107705 \%$ & $0,00137929 \%$ & $5,80391491 \%$ \\
\hline ITSA3 & N1 & $0,00004710 \%$ & $0,00004022 \%$ & $0,52317148 \%$ \\
\hline ITSA4 & N1 & $0,00101951 \%$ & $0,00123808 \%$ & $6,63730773 \%$ \\
\hline ITUB3 & N1 & 0,00090193\% & $0,00108980 \%$ & $5,96652434 \%$ \\
\hline ITUB4 & N1 & $0,00020257 \%$ & $0,00024066 \%$ & $1,49615751 \%$ \\
\hline BPNM4 & N1 & $0,00008023 \%$ & $0,00008216 \%$ & $0,80328706 \%$ \\
\hline PRBC4 & N1 & $0,00016364 \%$ & $0,00015301 \%$ & $1,66174823 \%$ \\
\hline RAPT3 & N1 & $0,00058830 \%$ & $0,00072260 \%$ & $3,66418338 \%$ \\
\hline RAPT4 & N1 & $0,00008550 \%$ & $0,00009310 \%$ & $0,78450287 \%$ \\
\hline
\end{tabular}


(continuação)

\begin{tabular}{|c|c|c|c|c|}
\hline Nome da ação & $\begin{array}{c}\text { Nível de } \\
\text { Gover- } \\
\text { nança }\end{array}$ & Retorno esperado & $\begin{array}{c}\text { Risco } \\
\text { Esperado }\end{array}$ & Participação \\
\hline SUZB5 & N1 & $0,00043105 \%$ & $0,00051723 \%$ & $2,96481311 \%$ \\
\hline USIM3 & N1 & $0,00104063 \%$ & $0,00137014 \%$ & $4,94610746 \%$ \\
\hline USIM5 & N1 & $0,00074185 \%$ & $0,00097075 \%$ & $3,60868759 \%$ \\
\hline VALE3 & N1 & $0,00054335 \%$ & $0,00066999 \%$ & $3,41091781 \%$ \\
\hline VALE5 & N1 & $0,00011415 \%$ & $0,00014044 \%$ & $0,75356800 \%$ \\
\hline ABCB4 & $\mathrm{N} 2$ & $0,00079642 \%$ & $0,00075857 \%$ & $6,25006489 \%$ \\
\hline DAYC4 & N2 & $0,00064176 \%$ & $0,00063342 \%$ & $7,00571653 \%$ \\
\hline FJTA4 & $\mathrm{N} 2$ & $0,00079956 \%$ & $0,00076681 \%$ & $6,74312452 \%$ \\
\hline GOLL4 & N2 & $0,00111625 \%$ & $0,00103119 \%$ & $5,90914998 \%$ \\
\hline IDVL4 & $\mathrm{N} 2$ & $0,00047838 \%$ & $0,00051299 \%$ & $8,84728533 \%$ \\
\hline POMO3 & N2 & $0,00076515 \%$ & $0,00074403 \%$ & $7,36032053 \%$ \\
\hline POMO4 & N2 & $0,00077149 \%$ & $0,00073408 \%$ & $5,91025118 \%$ \\
\hline MULT3 & N2 & $0,00066029 \%$ & $0,00066121 \%$ & $8,18164011 \%$ \\
\hline PINE4 & N2 & $0,00062887 \%$ & $0,00063019 \%$ & $7,86358669 \%$ \\
\hline SANB11 & $\mathrm{N} 2$ & $0,00079712 \%$ & $0,00076102 \%$ & $6,54169227 \%$ \\
\hline STBP11 & $\mathrm{N} 2$ & $0,00070708 \%$ & $0,00069415 \%$ & $7,20560435 \%$ \\
\hline SLED4 & $\mathrm{N} 2$ & $0,00071806 \%$ & $0,00070586 \%$ & $7,40595947 \%$ \\
\hline SFSA4 & $\mathrm{N} 2$ & $0,00068276 \%$ & $0,00066810 \%$ & $7,24229810 \%$ \\
\hline SULA11 & $\mathrm{N} 2$ & $0,00068582 \%$ & $0,00068097 \%$ & $7,53330813 \%$ \\
\hline BEEF3 & NM & $0,00070604 \%$ & $0,00002691 \%$ & $0,72821611 \%$ \\
\hline BEMA3 & NM & $0,00193302 \%$ & $0,00018721 \%$ & $5,06758323 \%$ \\
\hline BRFS3 & NM & $0,00050494 \%$ & $0,00002333 \%$ & $0,63132291 \%$ \\
\hline CARD3 & NM & $-0,00246265 \%$ & 0,00012017\% & $3,25199095 \%$ \\
\hline CIEL3 & NM & $0,00364605 \%$ & $0,00010669 \%$ & $2,88639426 \%$ \\
\hline CPFE3 & NM & $0,00152298 \%$ & $0,00015902 \%$ & $4,30750481 \%$ \\
\hline CSMG3 & NM & $0,00038318 \%$ & $0,00005072 \%$ & $1,37247802 \%$ \\
\hline CTIP3 & NM & $0,00072138 \%$ & $0,00003834 \%$ & $1,03749456 \%$ \\
\hline DASA3 & NM & $0,00016231 \%$ & $0,00002447 \%$ & $0,66227406 \%$ \\
\hline EMBR3 & NM & $0,00056735 \%$ & $0,00002283 \%$ & $0,61721380 \%$ \\
\hline ENBR3 & NM & $0,00060038 \%$ & $0,00007959 \%$ & $2,15180732 \%$ \\
\hline EQTL3 & NM & $0,00559392 \%$ & $0,00020948 \%$ & $5,66827297 \%$ \\
\hline ETER3 & NM & $0,00127151 \%$ & $0,00010732 \%$ & $2,90393952 \%$ \\
\hline FLRY3 & NM & $0,00068896 \%$ & $0,00011534 \%$ & $3,12066582 \%$ \\
\hline
\end{tabular}


(conclusão)

\begin{tabular}{lllll}
\hline Nome da ação & $\begin{array}{c}\text { Nível de } \\
\text { Gover- } \\
\text { nança }\end{array}$ & Retorno esperado & $\begin{array}{c}\text { Risco } \\
\text { Esperado }\end{array}$ & Participação \\
\hline GRND3 & NM & $0,00389485 \%$ & $0,00014985 \%$ & $4,05536112 \%$ \\
GSHP3 & NM & $0,00047818 \%$ & $0,00010064 \%$ & $2,72247728 \%$ \\
JBSS3 & NM & $0,00143256 \%$ & $0,00007079 \%$ & $1,91618635 \%$ \\
LIGT3 & NM & $0,00728761 \%$ & $0,00025950 \%$ & $7,03027931 \%$ \\
LLIS3 & NM & $0,00532638 \%$ & $0,00022880 \%$ & $6,19019200 \%$ \\
LOGN3 & NM & $-0,00024019 \%$ & $0,00003562 \%$ & $0,96235949 \%$ \\
MDIA3 & NM & $0,01658941 \%$ & $0,00044135 \%$ & $11,94838479 \%$ \\
NATU3 & NM & $-0,00031421 \%$ & $0,00015278 \%$ & $4,13325960 \%$ \\
ODPV3 & NM & $0,00223409 \%$ & $0,00008356 \%$ & $2,26138631 \%$ \\
PRVI3 & NM & $0,00098485 \%$ & $0,00010720 \%$ & $2,90317814 \%$ \\
TBLE3 & NM & $0,00763693 \%$ & $0,00038237 \%$ & $10,33835331 \%$ \\
TEMP3 & NM & $0,00179315 \%$ & $0,00005482 \%$ & $1,48445934 \%$ \\
TGMA3 & NM & $0,00038269 \%$ & $0,00009270 \%$ & $2,50441871 \%$ \\
TOTS3 & NM & $0,00131777 \%$ & $0,00004997 \%$ & $1,35303060 \%$ \\
TPIS3 & NM & $0,00117596 \%$ & $0,00013063 \%$ & $3,53361780 \%$ \\
WEGE3 & NM & $0,00148525 \%$ & $0,00008335 \%$ & $2,25589782 \%$ \\
\hline Fon $\%$ & & &
\end{tabular}

Fonte: os autores. 


\section{APÊNDICE B - CRITÉRIOS DE SELEÇÃO DOS MODELOS GARCH}

Tabela B.1 - Resumo empírico dos modelos GARCH das carteiras N1, N2 e NM

\begin{tabular}{|c|c|c|c|c|c|c|}
\hline \multirow[b]{2}{*}{ Modelo } & \multicolumn{2}{|c|}{ Carteira N1 } & \multicolumn{2}{|c|}{ Carteira N2 } & \multicolumn{2}{|c|}{ Carteira NM } \\
\hline & AIC & SBC & AIC & SBC & AIC & SBC \\
\hline $\begin{array}{l}\text { GARCH-M } \\
(1,1)\end{array}$ & 2.70172 & 2.72624 & 2.57419 & 2.59871 & 0.47556 & 0.50008 \\
\hline $\begin{array}{l}\text { GARCH-M } \\
(1,2)\end{array}$ & 2.70335 & 2.73278 & 2.57478 & 2.60421 & 0.48214 & 0.51156 \\
\hline $\begin{array}{l}\text { GARCH-M } \\
(2,1)\end{array}$ & 2.70346 & 2.73289 & 2.57551 & 2.60494 & 0.47753 & 0.51254 \\
\hline $\begin{array}{l}\text { GARCH-M } \\
(2,2)\end{array}$ & 2.70281 & 2.73714 & 2.57452 & 2.61431 & 0.48398 & 0.51831 \\
\hline
\end{tabular}

Fonte: os autores.

Tabela B.2 - Teste de ARCH-LM (10) para verificar presença de heterocedasticidade condicional

\begin{tabular}{lrr}
\hline \multicolumn{2}{c}{ Carteiras } & Probabilidade \\
\hline N1 & 2.01 & 0.84 \\
N2 & 17.53 & 0.06 \\
NM & 8.52 & 0.58 \\
\hline
\end{tabular}

Fonte: os autores.

\section{Como citar este artigo}

BESARRIA, Cássio Nóbrega et al. A qualidade das informações prestadas pelas empresas reduz os riscos de investimento? Uma análise empírica para os diferentes níveis de governança corporativa das empresas brasileiras. RACE, Revista de Administração, Contabilidade e Economia, Joaçaba: Ed. Unoesc, v. 14, n. 1, p. 11-378, jan./abr. 2015. Disponível em: < http://editora.unoesc.edu.br/index.php/ race $>$. Acesso em: dia/mês/ano.

Besarria, C. N., Paula, A. A., Araújo, B. S., Alves, J. N., Almeida, F. F., \& Monteiro, V. S. (2015). A qualidade das informações prestadas pelas empresas reduz os riscos de investimento? Uma análise empírica para os diferentes níveis de governançacorporativa das empresas brasileiras. RACE, Revista de Administração, Contabilidade e Economia, 14(1), 11-38. Recuperado em dia/mês/ano, de http:// editora.unoesc.edu.br/index.php/race 
